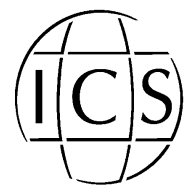

www.ics-elsevier.com

\title{
Lessons from biological processing of image texture
}

\author{
T. Maddess ${ }^{\mathrm{a}, *}$, Y. Nagai ${ }^{\mathrm{b}}$ \\ ${ }^{a}$ Centre for Visual Sciences, Australian National University, RSBS ANU, Canberra 0200, Australia \\ ${ }^{\mathrm{b}}$ Center for Information Science, Kokushikan University, Tokyo, Japan
}

\begin{abstract}
When designing artificial vision systems, it may be useful to examine the solutions 0.5 billion years of biological evolution have produced. Recent studies of human vision; studies of macaque visual cortical function; and behavioural studies of bee vision, all indicate that different species have evolved related approaches for discriminating image textures. This common strategy uses short-range 4th-order spatial correlations. Isotrigon textures, ensemble averages of which have 3 rd-order correlation functions that are equal to 0 , are useful for studying this sense. Recent results from humans and bees, and methods for producing new isotrigon textures are described. (C) 2004 Elsevier B.V. All rights reserved.
\end{abstract}

Keywords: Texture discrimination; Texture recognition; Isotrigon; Psychophysics; Bee vision

\section{Introduction}

Several modern signal discrimination methods have recently been shown to be related in terms of their tacit minimization of the sums of squares of 4th-order marginal cumulants [1]. Animals regularly make discriminations between mixtures of sensory inputs, and classify ad hoc categories of stimuli. Given the signal discrimination results, it would be surprising if our brains did not use 4th-order statistical measures to make discriminations. We describe the use and synthesis of classes of texture patterns, ensembles of which have average 3 rd-order correlation functions $(3 \mathrm{CF}$ ) that do not differ significantly from 0 . Noise textures also have zero $3 \mathrm{CFs}$, hence, the textures are said to be isotrigon with noise. Isotrigon patterns also have zero means and 2nd-order correlation functions. Thus, isotrigon textures can only be discriminated based on measures related to their 4th, or higher order, correlation functions [2]. Thus, the isotrigon textures provide a convenient way to test the sensitivity of humans and animals to 4th-order image structure. This paper presents data addressing the following points:

1. Can animals vastly unrelated to humans also discriminate isotrigon textures? If so the mechanisms selected by evolution might be robust and exploitable.

\footnotetext{
* Corresponding author. Tel.: +61-2-6125-4099; fax: +61-2-6125-3808.
}

E-mail address: ted.maddess@anu.edu.au (T. Maddess). 
A

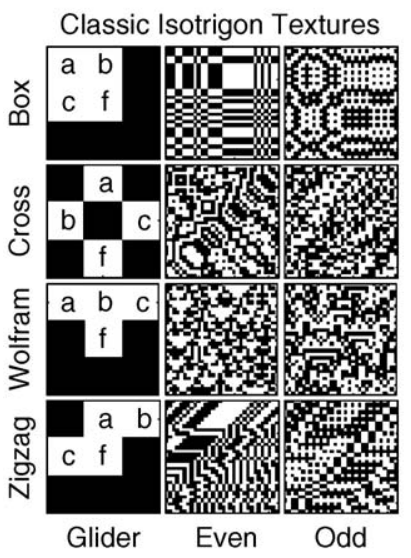

B

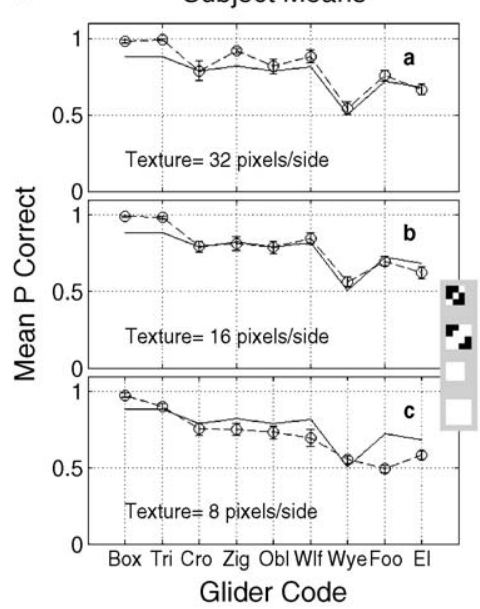

Fig. 1. (A) Binary isotrigon textures. Each texture matrix is initialized with dark $(-1)$ or bright (1) pixels $(p=0.5)$. The checks are then recoloured in by a recursion rule operating via a moving $3 \times 3$ pixel domain or glider (left column). The gliders contain three active pixels, $\mathrm{a}, \mathrm{b}, \mathrm{c}$. Two discrete equations: $f= \pm a b c$, govern the recursion producing the Even and Odd textures (right 2 columns). (B) Mean probability (dashed, symbols) of humans discriminating different isotrigon textures from each other (by glider) or from random patterns compared with results of a simple 4th-order model (solid lines) based on two scales of receptive fields (insert right).

2. How well can humans discriminate the currently available isotrigon textures?

3. What are the essential characteristics of any model that mimics human performance? (a) Multi-scalar analysis, if so how many scales? (b) Oriented detectors, if so how many orientations? (c) Fourth order, is it really required?

4. Can we generate more, well structured, isotrigon patterns for new experiments?

Studies using isotrigon patterns and, Visual Evoked Potentials [3], functional imaging with PET [4] and MRI [5,6], all provide evidence for human differential sensitivity to pattern structure defined by 4 th and higher correlations.

We have examined human pre-attentive (presentation $<250 \mathrm{~ms}$ ) texture discrimination [7]. Lengthy inspection of the textures of Fig. 1 reveals various features: triangles, crosses, etc. These features are less obvious when the textures are presented briefly. Pre-attentive mechanisms most likely represent early visual processing. In agreement with this idea, a majority of neurons in $\mathrm{V} 1$ of the macaque have been shown to be sensitive to 4th-order correlations [8]. Thus, sensitivity to higher order spatial correlations appears to be among the earliest information extracted by our visual brain.

\section{Methods}

The achromatic textures used here were first described by Victor and Conte [9]. We employed three octaves of textures sizes, from $8 \times 8$ to $32 \times 32$ pixels ( $29^{\prime}$ square). The stimulus was on at full contrast for $204 \mathrm{~ms}$. There were three types of trials in which subjects compared either Even vs. Random (binary) textures (ER), Odd vs. Random 
textures (OR), or Even vs. Odd textures (EO) [7]. For the bees, we used Even and Odd Box textures (Fig. 1, top row), and random textures. Stimuli were presented to honey bees in a Y-maze apparatus. Bees $(N=15)$ were rewarded with sugar-water if they flew to the positive patterns. Olfactory and other possible cues were eliminated. Patterns were exchanged every $8 \mathrm{~min}$ from a selection of training stimuli.

\section{Results and discussion}

Fig. 1B summarises psychophysical experiments [7] showing the mean probability of a correct discrimination (circles and dashed lines) for three texture sizes (rows). The plotted functions are the averages for ER, OR and EO data. The patterns of Fig. 1A were well discriminated. Two textures, Wye and Foot (not shown) were poorly discriminated. Similar data had previously been reported using a quite different methodology [9]. The solid curve is the output of a quadratic discriminate model (Fig. 2A, caption). The probabilities obtained from the model are unscaled, but show remarkable similarity to the data. The discriminant model used just four receptive field types (Fig. 2A, left).

Fig. 2B (upper panel) illustrates the results of training bees to discriminate Odd from Even Box textures [10]. Following training and testing with different sets of Even and Odd textures, the same bees were confronted with sets of Odd vs. Random textures, with the Odd patterns being rewarded (Fig. 2B, lower panel). The bees could distinguish these too
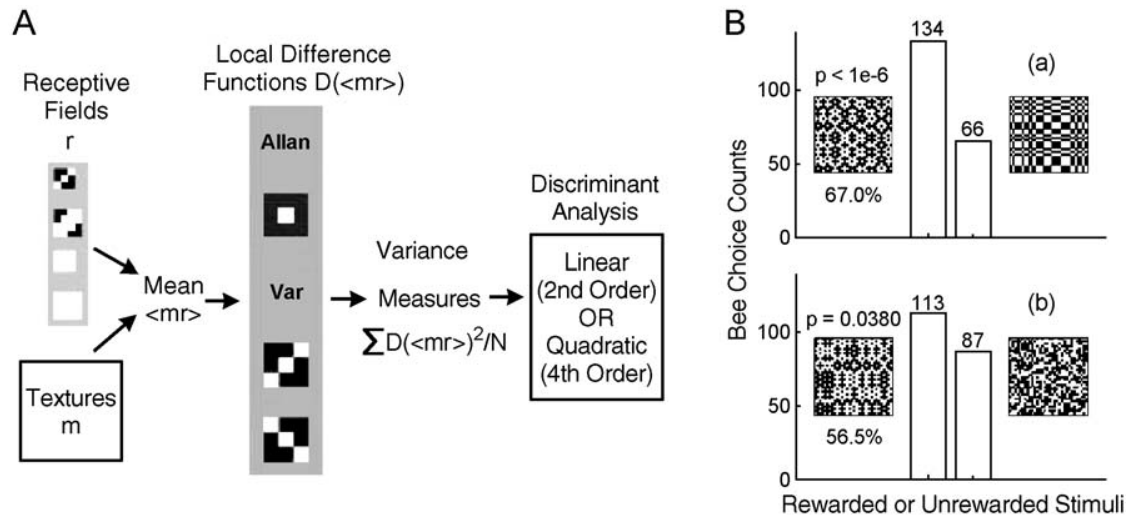

Fig. 2. (A) Diagrammatic version of the discrimination models examined. Four receptive fields (RFs) are illustrated in the strip at left. Five models then examined: (1) the global variance in these mean RF outputs $\left\langle m_{i} r_{i}\right\rangle$; (2) the Allan Variance, $\mathrm{AV}=1 / 2 \Sigma\left(\left\langle m_{i+1} r_{i+1}\right\rangle-\left\langle m_{i} r_{i}\right\rangle\right) 2 / N$, and three local versions of the AV. These comprised an unoriented case (UnO) and two oriented cases (Or1, Or2). UnO, Or1, and Or2 are illustrated in the central bar of A, where each check represents a portion of visual space the size of an RF. Here instead of simply taking the mean squared differences between $\left\langle m_{i} r_{i}\right\rangle$ we took instead weighted 2D differences, squared these and took their mean. Thus, the difference term in the AV is replaced by a local 2D differencing operation $D\left(\left\langle m_{i} r_{i}\right\rangle\right)$ to give $\Sigma D\left(\left\langle m_{i} r_{i}\right\rangle\right)^{2} / N$. Linear and Quadratic discriminant models based on these five variance measures were compared. The linear (2nd-order) models did not work. Oriented local variance was best. (B) Bee choice data following training with a set of Even and Odd isotrigon patterns. Each $32^{2}$ pixel pattern subtended $52^{\circ}$, bringing the characteristic spatial frequency, 0.31 cycles $/^{\circ}$, well within bee visual acuity. The upper panel shows discrimination rates for Even vs. Odd pattern, lower panel subsequent, untrained, discrimination of Even vs. Random patterns. Correct choices are shown by the left bar of each panel. 
without training $(p=0.038)$. This result was highly reproducible. We have subsequently demonstrated neurons in the bee brain [11] that have all the properties of a texture discrimination model proposed by Victor and Conte [9].

To obtain a better understanding of the neural mechanisms of human texture discrimination, which probably has quite a high dimensionality, we need a larger palette of isotrigon textures. We have examined [12] an alternative approach to generating textures involving the general discrete function governing the recursion process. For binary textures this function is $f(a, b, c)=x_{1}+x_{2} a+x_{3} b+x_{4} c+x_{5} a b+x_{6} b c+x_{7} a c+x_{8} a b c$, of which the Odd and Even texture discrete equations (Fig. 1A, legend) are special cases. We also explored the case for ternary textures, composed of $-1,0$ and 1 , where the discrete equation is a multinomial function of 27 terms producing $3^{27}=7.62 \times 10^{12}$ possible textures. We have demonstrated an abundance of three-level isotrigon textures [12].

We addressed the four points raised in Introduction. Very different animals have adopted similar texture discrimination strategies. The essential features of human discrimination included short-range analysis at about two scales, and at least two RF orientations [7]. Methods for generating new isotrigon patterns were discussed [12]. These new patterns might provide better resolution of the mechanisms of human texture discrimination. We have also given a plausible neurophysiological mechanism for computing the underlying 4th-order correlation measures [7].

\section{References}

[1] T.-W. Lee, et al., A unifying information-theoretic framework for independent component analysis, Comput. Math. Appl. 31 (2000) 1-21.

[2] J.D. Victor, Images, statistics and textures: implications of triple correlation uniqueness for texture statistics and the Julesz conjecture: comment, J. Opt. Soc. Am. A11 (1994) 1680-1684.

[3] J.D. Victor, M.M. Conte, Cortical interactions in texture processing: scale and dynamics, Vis. Neurosci. 2 (1989) 297-313.

[4] L.L. Beason-Held, et al., PET reveals occipitotemporal pathway activation during elementary form perception in humans, Vis. Neurosci. 15 (3) (1998) 503-510.

[5] L.L. Beason-Held, et al., Cortical regions involved in visual texture perception: a fMRI study, Brain Res., Cogn. Brain Res. 7 (2) (1998) 111-118.

[6] L.L. Beason-Held, et al., Striate cortex in humans demonstrates the relationship between activation and variations in visual form, Exp. Brain Res. 130 (2) (2000) 221-226.

[7] T. Maddess, Y. Nagai, Discriminating isotrigon textures, Vis. Res. 41 (2001) 3837-3860.

[8] K.P. Purpura, J.D. Victor, E. Katz, Striate cortex extracts higher-order spatial correlations from visual textures, Proc. Natl. Acad. Sci. U. S. A. 91 (18) (1994) 8482-8486.

[9] J.D. Victor, M.M. Conte, Spatial organization of nonlinear interactions in form perception, Vis. Res. 31 (1991) 1457-1488.

[10] T. Maddess, M. Davey, E. Yang, Discrimination of complex textures by bees, J. Comp. Physiol., A Sens. Neural Behav. Physiol. 184 (1999) 107-117.

[11] E.C. Yang, T. Maddess, Orientation-sensitive neurons in the brain of the honeybee (Apis mellifera), J. Insect Physiol. 43 (1997) 329-336.

[12] T. Maddess, et al., Binary and ternary textures containing higher order spatial correlations, Vis. Res. 44 (2004) $1093-1113$. 